\title{
Reducing the effective dose of cisplatin using gold nanoparticles as carriers
}

\author{
M. A. González-López ${ }^{1 *}$, E. M. Gutiérrez-Cárdenas², C. Sánchez-Cruz ${ }^{5}$, J. F. Hernández-Paz³ I. Pérez ${ }^{4}$ \\ J. J. Olivares-Trejo ${ }^{5}$ and O. Hernández-González ${ }^{6}$
}

*Correspondence:

marconygfb@yahoo.com.mx

${ }^{1}$ Unidad de Oncología

Torácica., CONACYT-Instituto

Nacional de Cancerología,

Cátedra-CONACYT, Avenida

San Fernando 22, Col.

Belisario Domínguez Secc.

16, 14080 Ciudad de México,

México

Full list of author information is available at the end of the article

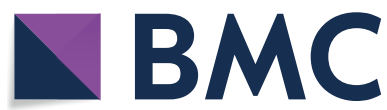

(C) The Author(s) 2020. This article is licensed under a Creative Commons Attribution 4.0 International License, which permits use, sharing, adaptation, distribution and reproduction in any medium or format, as long as you give appropriate credit to the original author(s) and the source, provide a link to the Creative Commons licence, and indicate if changes were made. The images or other third party material in this article are included in the article's Creative Commons licence, unless indicated otherwise in a credit line to the material. If material is not included in the article's Creative Commons licence and your intended use is not permitted by statutory regulation or exceeds the permitted use, you will need to obtain permission directly from the copyright holder. To view a copy of this licence, visit http://creativecommons.org/ licenses/by/4.0/. The Creative Commons Public Domain Dedication waiver (http://creativecommons.org/publicdomain/zero/1.0/) applies to the data made available in this article, unless otherwise stated in a credit line to the data.

\section{Background}

The increasing rate of cancer cases is alarming. In 2018, 9.6 million people died of the disease (WHO 2020). Current treatments, such as chemical therapy using cisplatin (CisPt) as a basis, cause considerable collateral damage to the patient (Ahmad 2017).

CisPt acts as an alkylating agent with affinity to DNA, binds to the guanine and cytosine residues, and prevents the processes of replication and transcription of the genetic material, resulting in cell death by apoptosis (Ahmad 2017). However, cisplatin is unable to differentiate between normal and cancerous cells. Moreover, cancer cells can develop 
resistance to it (Johnstone et al. 2016). This anticarcinogen also has serious side effects such as hair loss, weakness and pain (Astolfi et al. 2013).

Given this situation, it is necessary to seek new treatment alternatives that reduce or eliminate side effects. To achieve this, it is desirable to administer controlled doses, focused exclusively on attacking cancer cells.

The field of nanotechnology may offer an alternative way to treat cancer. Nanoparticles are particles with sizes between 1 and $100 \mathrm{~nm}$ (López 2013). They have various uses in nanomedicine, biosignalization, biological separation, molecular imaging and cancer therapy (Kaushik et al. 2009; Kuo et al. 2009; Ortiz-Benitez et al. 2015; Sánchez et al. 1996). It is possible to engineer the nanoparticles to deliver drugs to specific cells or parts of the human body, although some of them can become, due to their atomic components, toxic, non-selective, and unstable (Ma et al. 2015; Nath et al. 2017). One of the nanoparticles that does not suffer from these shortcomings is the gold nanoparticle (AuNps) which combines ease of synthesis, chemical stability, and limited cytotoxic effects (Couto et al. 2016). Thus, it has been used in signaling, in imaging and as a drug carrier, given its low toxicity (Alkilany and Murphy 2010; Craig et al. 2012).

The objective of this research is to synthesize AuNps, functionalize them with CisPt, and release them into the cancer cells. In this study, we suggest a methodology for the functionalization of the AuNps and carry out in vitro experiments with several concentrations of CisPt to assess their cytotoxic effect. For this purpose, we obtained two types of functionalized gold nanoparticles, one with polyethylene glycol (PEG), henceforth AuNpPEG, and another with PEG and CisPt, henceforth AuNpPEGCisPt. We carried out chemical analysis based on energy dispersion spectroscopy (EDS) using scanning electron microscopy to verify the core-shell type of our samples. To check chemical bonding, we also used Fourier transform infrared spectroscopy (FTIR). We measured the size of the nanoparticles using an electronic transmission microscope (TEM) and their hydrodynamic size using dynamic light scattering (DLS) and potential $Z$. The tests against cancer cell lines showed that the AuNpPEGCisPt reaches inhibitory concentration $50\left(\mathrm{IC}_{50}\right)$ using a smaller dose than the CisPt by itself. This may be the first step to achieving an alternative therapy for cancer, using nanotechnology.

The present investigation seeks to develop a nanoparticle that would deliver the smallest effective quantity of cisplatin to the cancer-affected area.

\section{Results}

\section{The functionalization and synthesis of gold nanoparticles}

The AuNps possess a characteristic spectrum when visualized in the UV/VIS spectrophotometer (Ji et al. 2007). In this case, the AuNps had a peak at $519 \mathrm{~nm}$, AuNpPEG at $523 \mathrm{~nm}$ and AuNpPEGCisPt at $525 \mathrm{~nm}$ (Fig. 1). We used TEM to estimate the size and shape of the different nanoparticles, showing that the AuNps have a diameter of $8.6 \pm 1.97 \mathrm{~nm}$, AuNpPEG of $9.5 \pm 1.24 \mathrm{~nm}$ and AuNpPEGCisPt of $10 \pm 1.76 \mathrm{~nm}$. All the nanoparticles synthesized have a spherical shape (Fig. 2). In addition, we determined the hydrodynamic size, $Z$ potential and polydispersity index of the Nps (Table 1); Nps, being immersed either in $\mathrm{H}_{2} \mathrm{O}$ and DMEM medium without fetal bovine serum, tended to form agglomerates, and $Z$ potential was use to $1 \mathrm{mV}$. On the other hand, when the nanoparticles were immersed in DMEM medium supplemented with fetal bovine serum 


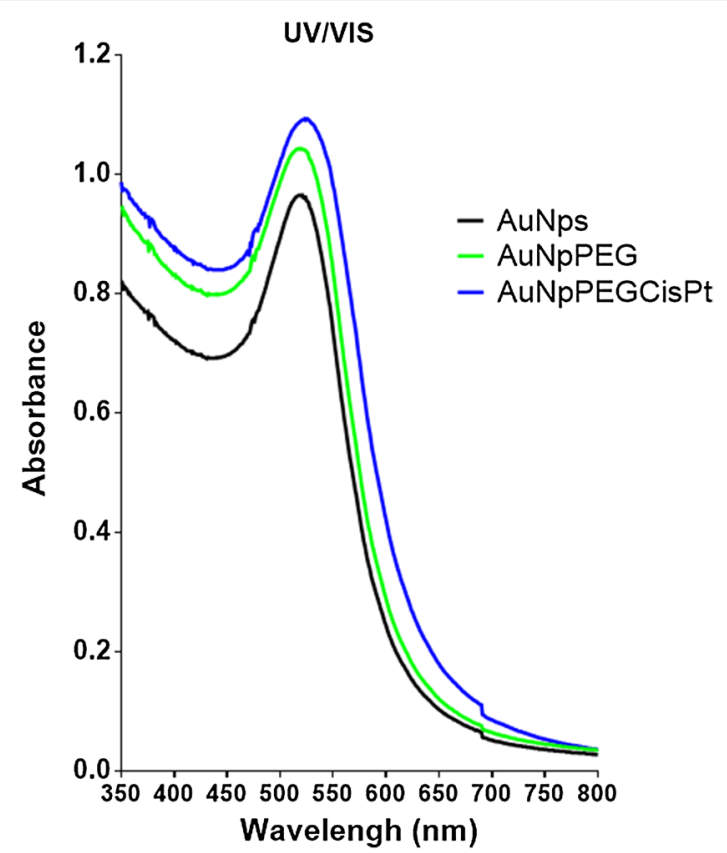

Fig. 1 UVNIS Ultraviolate-visible spectroscopy spectrum of the synthesized nanoparticles

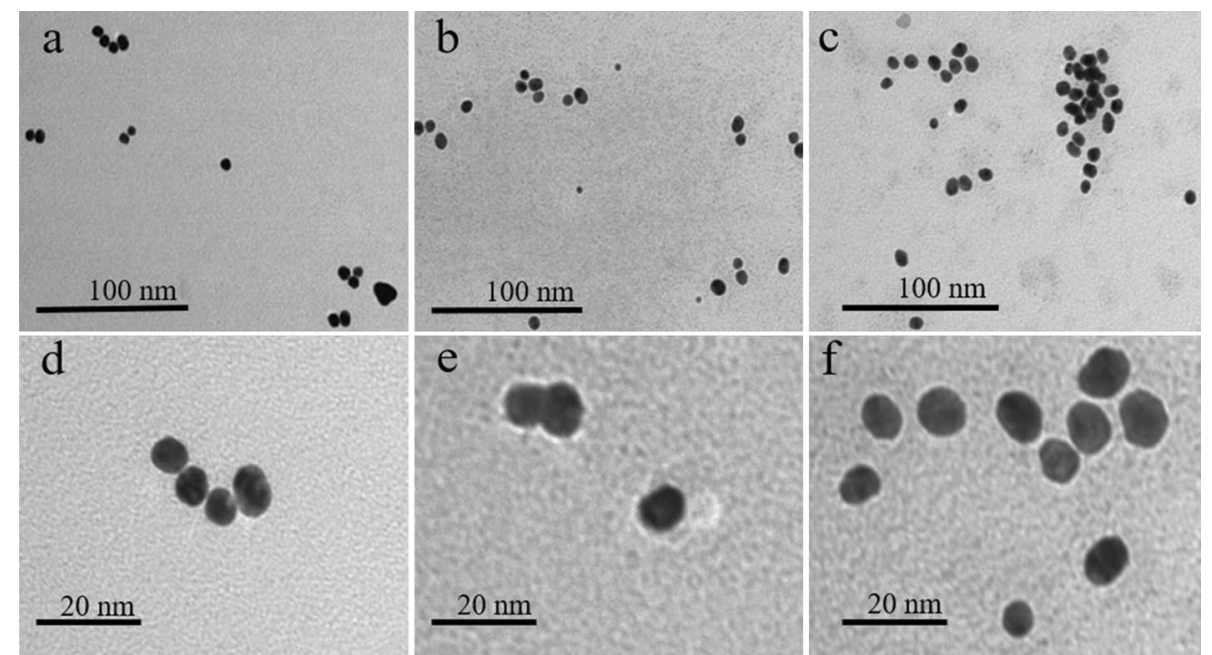

Fig. 2 TEM micrographs of the different nanoparticles synthesized. a, d AuNps; b, e AuNpPEG; c, $\mathbf{f}$ AuNpPEGCisPt. a-c $\times 65000, \mathbf{d}-\mathbf{f} \times 210000$

at $10 \%$, they also tended to form agglomerates although of smaller size, $Z$ potential being close to $1 \mathrm{mV}$.

\section{Functioning of the AuNps with PEG and CisPt}

One aim of this investigation was to bond the CisPt to the AuNps. Direct bonding resulted in the product obtained precipitating and forming large agglomerates. We used PEG as an agent to solubilize and stabilize the bond (Vijaya and Ganesan 2011). We 
Table 1 Hydrodynamic characteristics of the Nps synthesized in different solutions

\begin{tabular}{lccc}
\hline Nanoparticles & Size $(\mathbf{n m}) \pm$ SD & PDI \pm SD & Zeta potential $(\mathbf{m V}) \pm$ SD \\
\hline AuNps $\left(\mathrm{H}_{2} \mathrm{O}\right)$ & $371.3 \pm 144.6$ & $0.566 \pm 0.290$ & $0.826 \pm 0.641$ \\
AuNpPEG $\left(\mathrm{H}_{2} \mathrm{O}\right)$ & $149.5 \pm 16.61$ & $0.219 \pm 0.008$ & $-1.23 \pm 0.218$ \\
AuNpPEGCisPt $\left(\mathrm{H}_{2} \mathrm{O}\right)$ & $594.1 \pm 323.8$ & $0.623 \pm 0.326$ & $0.738 \pm 0.803$ \\
AuNps (w/o serum) & $1942 \pm 402.9$ & $0.992 \pm 0.014$ & $0.172 \pm 0.606$ \\
AuNpPEG (w/o serum) & $101.6 \pm 40.97$ & $0.197 \pm 0.042$ & $-0.007 \pm 0.012$ \\
AuNpPEGCisPt (w/o serum) & $147.7 \pm 114.1$ & $0.447 \pm 0.201$ & $-0.0097 \pm 0.008$ \\
AuNps (serum) & $55.12 \pm 5.023$ & $0.650 \pm 0.11$ & $0.044 \pm 0.099$ \\
AuNpPEG (serum) & $49.08 \pm 0.51$ & $0.531 \pm 0.006$ & $-0.014 \pm 0.017$ \\
AuNpPEGCisPt (serum) & $67.52 \pm 31.78$ & $0.344 \pm 0.131$ & $-0.078 \pm 0.06$ \\
\hline
\end{tabular}

attempted to join the CisPt to the AuNps and the modified technique reported by Sun et al. (2014) is the one that gave better results. We analyzed each synthesized nanoparticle to determine the type of bonding created between the CisPt and the AuNps. For this purpose, we used the FTIR technique. We placed the samples in aqueous solution. $\mathrm{H}_{2} \mathrm{O}$ produces a large signal between 3300 and $3500 \mathrm{~cm}^{-1}$ where hydroxyls groups occur (Botha et al. 2015). The AuNps show a characteristic spectrum of this type when they are in aqueous solution (Botha et al. 2015). When we functionalized the AuNps with PEG, we observed the $\mathrm{C}=\mathrm{O}$ bonds, proper to this polymer (at 1634 and $1633 \mathrm{~cm}^{-1}$ ). In addition, we found a peak corresponding to $\mathrm{CH}$ groups of PEG at $2152 \mathrm{~cm}^{-1}$ (Ha et al. 2005). Hydrogen bonding, tight $\mathrm{OH}$ junctions between 3570 and $3200 \mathrm{~cm}^{-1}$, and wide junctions of $\mathrm{H}$ between 3400 and $3200 \mathrm{~cm}^{-1}$ indicate that the CisPt and the different nanoparticles are in interaction with $\mathrm{H}_{2} \mathrm{O}$ (Botha et al. 2015). Finally, we observed Pt-N bonds in AuNpPEGCisPt, corresponding to CisPt; the signals occurred at 3945, 3458, 3418, 615, $581 \mathrm{~cm}^{-1}$ (Rajath et al. 2015), 599 and $560 \mathrm{~cm}^{-1}$ (Balice and Theophanides 1970) (Fig. 3).

\section{The bonding of CisPt with AuNps}

To confirm that the CisPt had coupled with the AuNps, and the type of bond which existed, we carried out an analysis based on SEM/EDS and FTIR. Moreover, we used the EDS analysis to verify the composition of the AuNps and we took this as a point of reference to compare the functionalized nanoparticles with PEG (Fig. 4b) and CisPt. The AuNps showed the presence of $\mathrm{Au}, \mathrm{O}$ and $\mathrm{Ca}$ (Fig. 4a). When analyzing the AuNpPEGCisPt, we observed the presence of the chemical components of the CisPt, such as $\mathrm{Pt}, \mathrm{Cl}$ and $\mathrm{N}$, in addition to Au (Fig. 4c). Using the FTIR technique, we detected the elements which make up the AuNpPEGCisPt nanoparticle and the links which form between these elements as we explained in the previous paragraph.

\section{Cytotoxic effect of nanoparticles}

To determine the cytotoxic effect of the different nanoparticles synthesized, it was necessary to challenge them against the cervical cancer (HeLa), gastric cancer (AGS) and glioblastoma (C6) cell lines. In the same way, we tested the CisPt as a positive control. This investigation suggests that neither AuNps (open square) nor AuNpPEG 


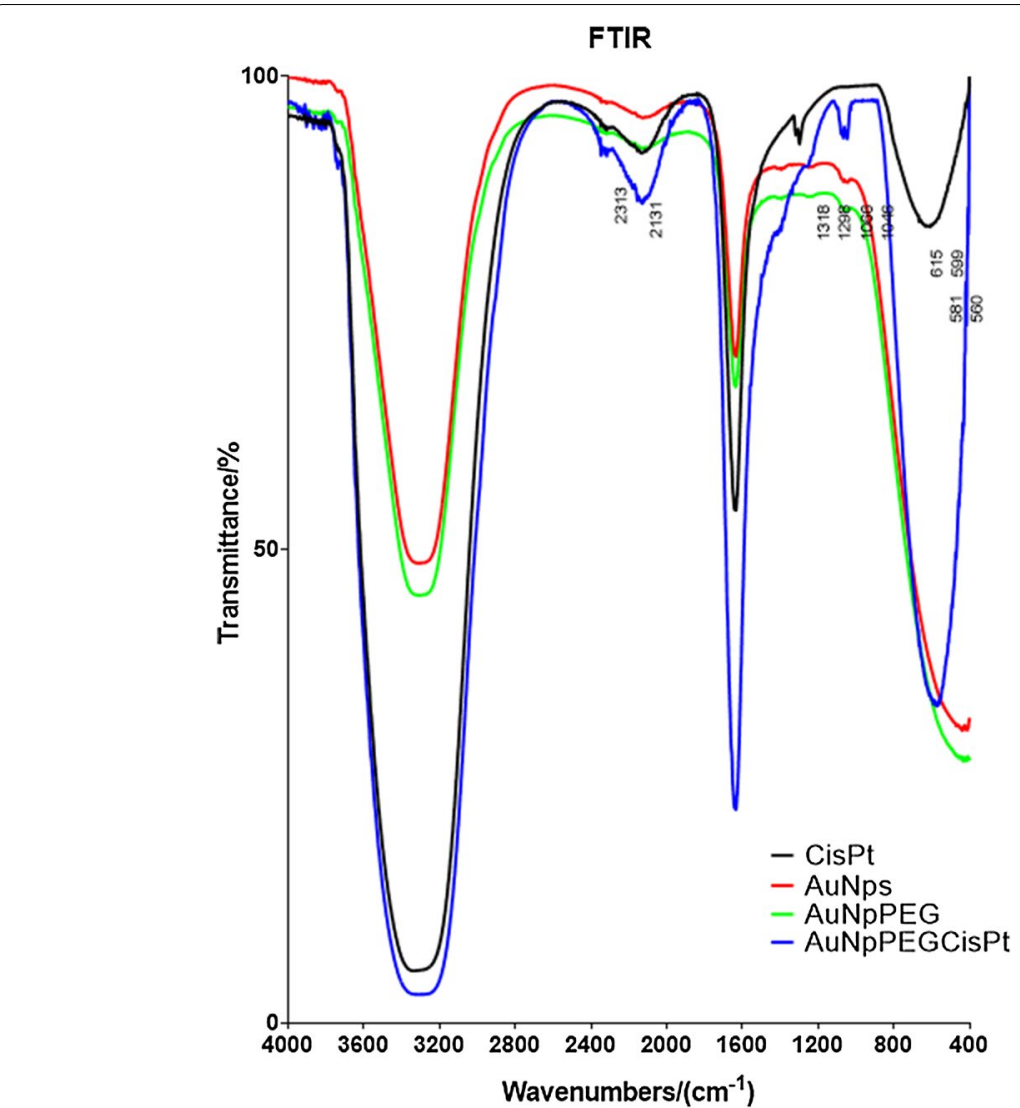

Fig. 3 FTIR spectrum of CisPt and of the nanoparticles synthesized. AuNps, AuNpPEG and AuNpPEGCisPt. The numbers indicate the peaks where the CisPt occurs and their interactions with the AuNpPEGCisPt

(open triangle) has a cytotoxic effect on the different cancer cell lines, or that this is minimal (Fig. 5a-c).

On the one hand, CisPt (open circle) causes $\mathrm{IC}_{50}$ at a concentration of $69.2 \pm 3.2 \mu \mathrm{g} /$ $\mathrm{mL}$ in HeLa cells, $286.5 \pm 2.5 \mu \mathrm{g} / \mathrm{mL}$ in AGS cells and $379.3 \pm 3.0 \mu \mathrm{g} / \mathrm{mL}$ in C6 cells. On the other hand, AuNpPEGCisPt (open diamond) causes an $\mathrm{IC}_{50}$ in HeLa cells at $104.5 \pm 7.0 \mu \mathrm{g} / \mathrm{mL}$, in AGS cells at $76.5 \pm 5.0 \mu \mathrm{g} / \mathrm{mL}$ and in C6 cells at $97.2 \pm 5.4 \mu \mathrm{g} /$ $\mathrm{mL}$ (Fig. 5, Table 2).

\section{Discussion}

CisPt is widely used in cancer therapy, sometimes in combination with other drugs, despite its multiple side effects. Nanotechnology provides an alternative solution that unites the effectiveness of CisPt and a reduction of its secondary effects.

In this study, we carried out the synthesis of a nanoparticle using the Turkevich et al. (1951) technique. We obtained AuNps functionalized with PEG and CisPt. The AuNps synthesized exhibits a characteristic spectrum in solution (Alcantar et al. 2000).

The first step out was to determine the composition of the nanoparticles. We used the FTIR technique that indicates the presence of the elements that make up each of 

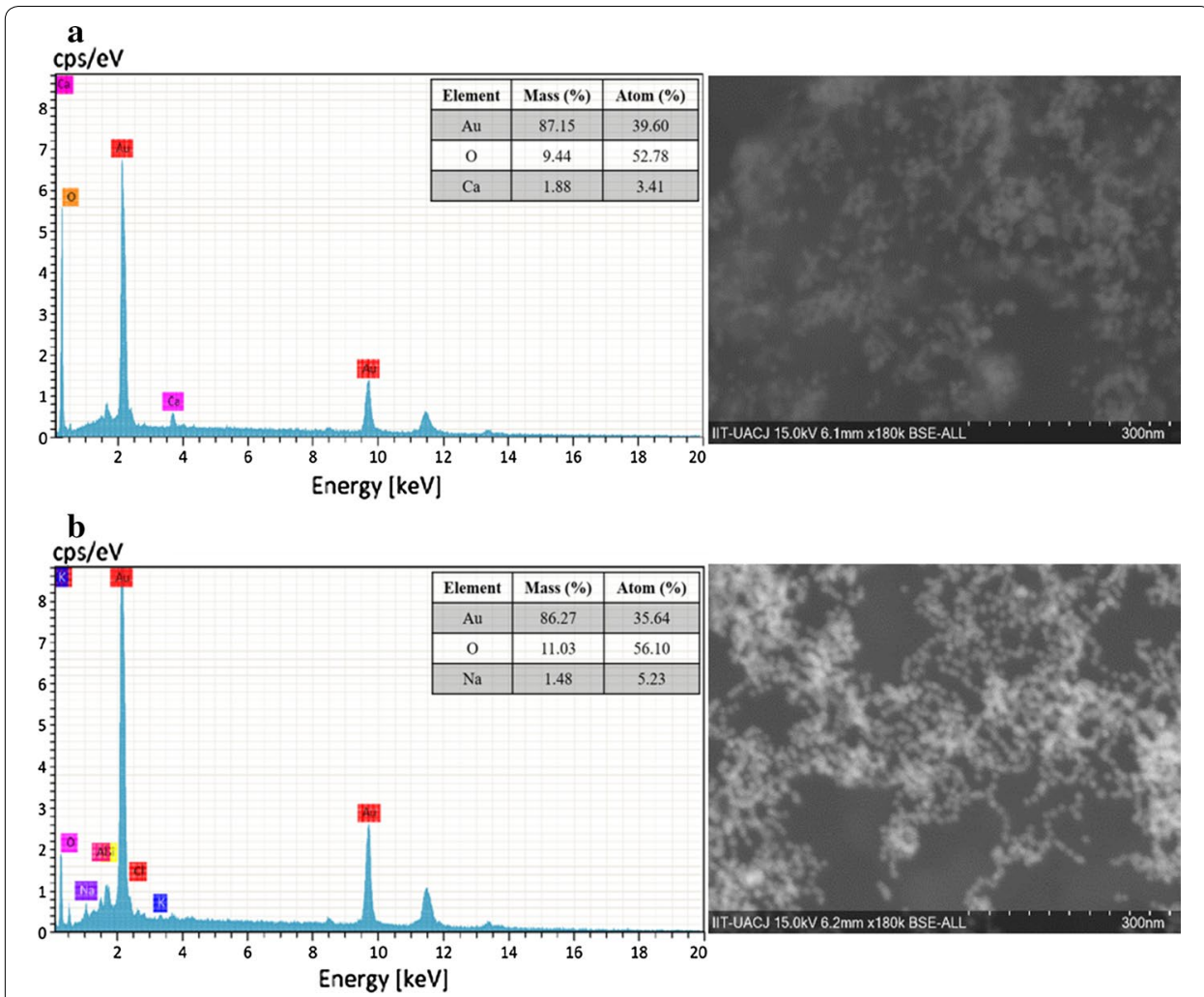

c

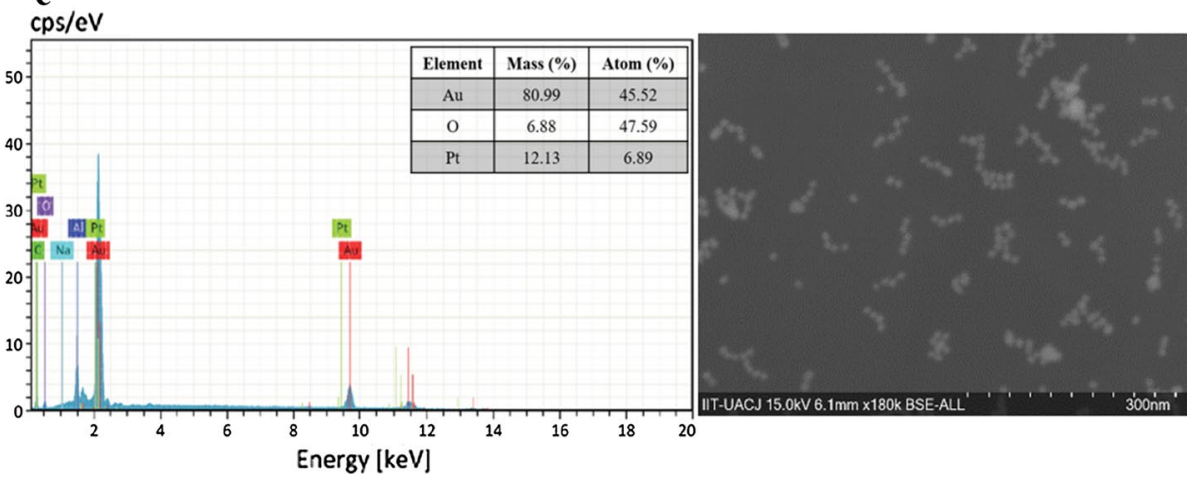

Fig. 4 Energy dispersive spectroscopy, atomic concentration and scanning electron microscopy: a AuNps, b AuNpPEG and c AuNpPEGCisPt. Micrographs $\times 180$

the synthesized Nps by recognizing the bonds they have formed. The EDS technique was then used to determine the composition of the Nps quantitatively.

We observed two signals using the FTIR analysis: one in the range between 2900 and $3800 \mathrm{~cm}^{-1}$ and another between 1600 and $1700 \mathrm{~cm}^{-1}$. It seems that the $\mathrm{OH}$ signal overshadowed the methyl groups that the $\mathrm{OH}$ groups produce (Botha et al. 2015). When functionalizing the AuNps with PEG, the methyl groups $\left(2960-2870 \mathrm{~cm}^{-1}\right.$ ) and carboxylic acid (3400-3200 $\mathrm{cm}^{-1}$ ), the $\mathrm{H}_{2} \mathrm{O}$ signal overshadowed them (Botha et al. 2015). In the case of the CisPt adhered to the AuNpPEG, the bonds Pt-N (599-560 $\left.\mathrm{cm}^{-1}\right)$ and Pt (3458-3418, 1641-1640 and 599-560 $\mathrm{cm}^{-1}$ ) also showed this effect (Rajath et al. 2015). CisPt displays two signals at 1318 and $1298 \mathrm{~cm}^{-1}$. We also 


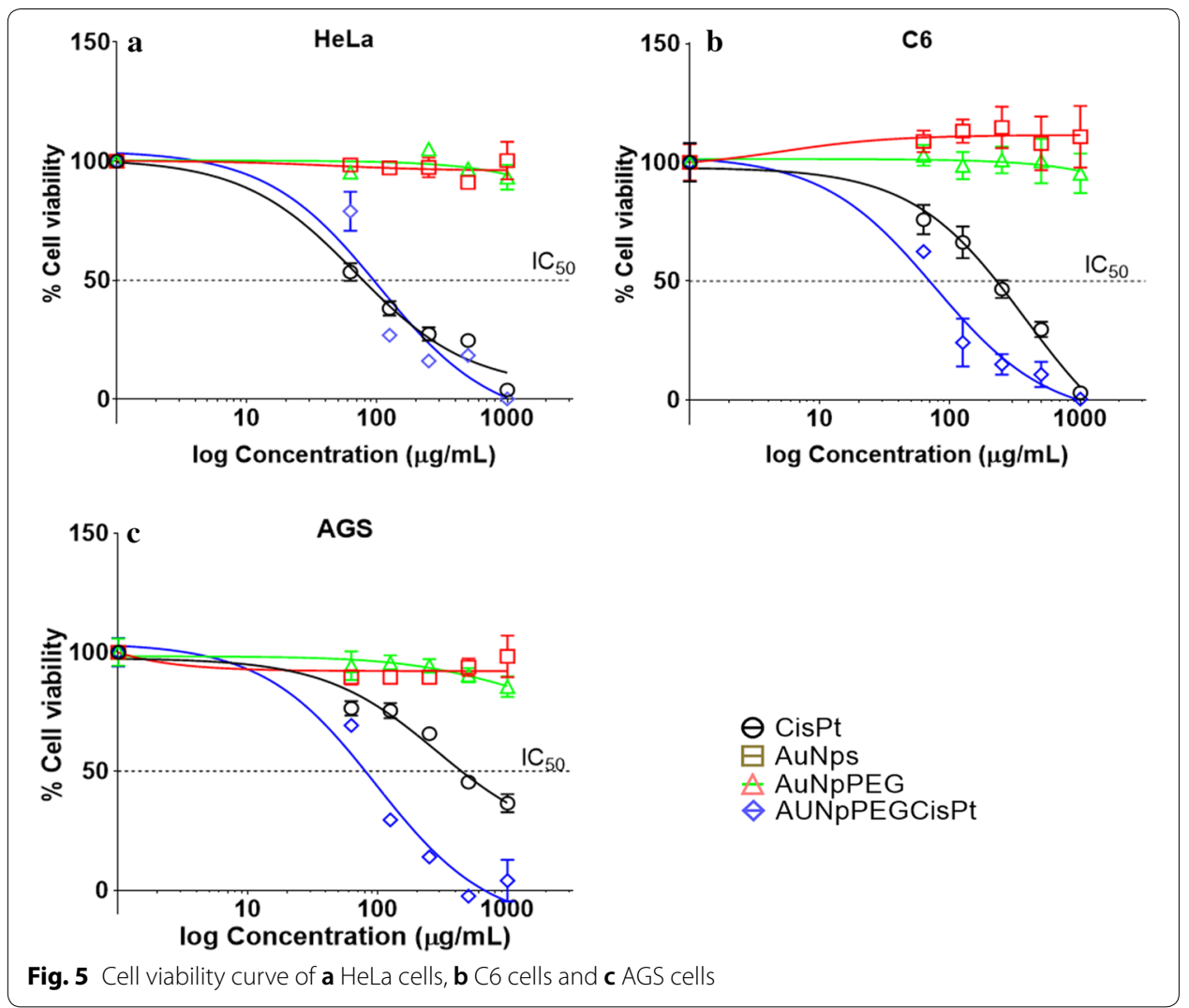

Table 2 The quantity of CisPt and AuNpPEGCisPt which causes $I_{50}$ in the different cell lines

\begin{tabular}{|c|c|c|c|}
\hline Cell line & CisPt $\left(I C_{50} \mu \mathrm{g} / \mathrm{mL}\right)$ & $\begin{array}{l}\text { AuNpsPEGCisPt ( }\left(\mathrm{C}_{50} \mu \mathrm{g} /\right. \\
\mathrm{mL} \text { ) }\end{array}$ & $\begin{array}{l}\text { CisPt } \\
\text { inside the AuNpPEGCisPt } \\
(\mu \mathrm{g} / \mathrm{mL})\end{array}$ \\
\hline HeLa & $69.2 \pm 3.2$ & $104.5 \pm 7.0$ & 7.31 \\
\hline C6 & $379.3 \pm 3.0$ & $76.5 \pm 5.0$ & 5.35 \\
\hline AGS & $286.5 \pm 2.5$ & $97.2 \pm 5.4$ & 6.80 \\
\hline
\end{tabular}

observed these signals in AuNpPEGCisPt at 1066 and $1046 \mathrm{~cm}^{-1}$. A possible explanation for this difference between the peaks is the existence of an alteration of the ions of the CisPt, which encapsulate inside the matrix of the AuNpPEGCisPt (Rajath et al. 2015).

To confirm directly the functionalization of the AuNps with PEG and CisPt, we used scanning electron microscopy and transmission electron microscopy. The micrographics (SEM/EDS) corresponding to the three nanoparticles are given in Fig. 4. We stress that we took the images in the backscattering electron mode to detect any visual difference in the atomic composition, with the aim of exposing the core-shell shape. The images are blurred and remained so, even using a higher resolution, because the size of the sample was very small. Therefore, to check any possible bonding between AuNps and PEG/CisPt, we carried out an EDS analysis in the punctual mode, that is, and an 
EDS at a point in the image. For the case of AuNps, we carried out punctual EDS measurements on several AuNPs. The results showed a high percentage of the nanoparticle is $\mathrm{Au}$ (Fig. $4 \mathrm{a}-\mathrm{C}$ ). By a similar procedure, we analyzed the samples with AuNpPEGCisPt and we detected traces of $\mathrm{Au}, \mathrm{Pt}$ and $\mathrm{O}$ (Fig. 4c). This is what one would expect if PEG and CisPt invariably bonded to AuNps. To rule out the possibility that these components appeared all over the substrate, we carried out several punctual EDS in the interstitial regions between nanoparticles and we observed no traces of $\mathrm{Au}, \mathrm{Pt}, \mathrm{Cl}, \mathrm{Na}$, or $\mathrm{O}$. This shows that, in between the nanoparticle areas, there is no other material. Therefore, it is possible to deduce that PEG and CisPt bond to AuNps.

Once we have found the composition of each of the synthesized Nps, we proceed to measure its size. It is important to note that the size of the Nps is influenced by the characteristics of the environment in which they are located (Moore et al. 2015; Wang et al. 2015). That is why we measured the size of the different Nps after their synthesis and then their size in conditions closer to their physiological conditions. That is, their hydrodynamic size and potential $Z$ were determined. Both parameters gave us an idea of how Nps would behave in a more complex system such as cancer cell lines (Lowry et al. 2016).

The size of the Nps after their synthesis was measured by TEM. It was found to be $8.6 \mathrm{~nm}$ with a standard deviation of $1.97 \mathrm{~nm}$ for AuNps, and $10 \mathrm{~nm}$ with standard deviation of $1.76 \mathrm{~nm}$ for AuNpPEGCisPt. This clearly shows that there is an overall increase in diameter of $1.4 \mathrm{~nm}$ which can only be attributed to the functionalization of the AuNps with PEG and CisPt. Gathering all this information, we could make a rough estimate of the quantity of CisPt that adhered to the AuNps. We subtracted the volume of the sphere of AuNps from the volume of AuNpPEGCisPt, which is the volume occupied by the "shell" full of PEG and CisPt. To determine the quantity of CisPt in this shell, we divide the volume of the shell by the volume of a CisPt molecule and multiply it by 7\% which is the maximum atomic concentration of Pt in AuNpPEGCisPt (according to the EDS). Taking the radius of a single CisPt molecule as $0.5 \mathrm{~nm}$ (Baowan et al. 2017), we found that each AuNpPEGCisPt has roughly 462 molecules of CisPt, which corresponds to a mass of $2.263 \times 10^{-19} \mathrm{~g}$.

As for the hydrodynamic size and potential $Z$, we have confirmed that the behavior of the different Nps is influenced by the medium in which they are resuspended (Danaei et al. 2018). We observed that the AuNp, regardless of the medium in which they are resuspended, tend to form agglomerates. This is one of the reasons why we decided to functionalize the AuNps with PEG. Previous work shows that PEG is a compound that provides solubility and stability to the AuNps (Guerrini et al. 2018). In our case, we observed that, when functionalizing the AuNps with PEG, the size of the agglomerates which formed decreases.

Finally, the behavior of the Nps was observed in an identical medium to that in which the cancer cells develop. Under these conditions, closer to their physiological conditions, we observed that the agglomerates were smaller and more stable in a medium similar to the conditions found in cellular development.

Once we had confirmed that the AuNpPEGCisPt contained CisPt and that its behavior in a physiological-like environment was acceptable, we proceeded to perform cell viability tests to determine whether the synthesized Nps had a cytotoxic 
effect on cancer cell lines. First, it was necessary to establish if, in carrying out the feasibility test, there was no overlap (and subsequent overestimation) of the wavelengths of the product of MTT metabolism (formazan) and the wavelengths of the cells and the different synthesized Nps. Our results show (Additional file 1) that there is no overlap between the wavelengths of the cells, the Nps and the formazan. Hence, we did not obtain overestimated figures when determining the cell viability and the $\mathrm{IC}_{50}$. It has been shown that AuNps and functionalized AuNps do not interfere with the MTT feasibility tests. (Altunbek and Culha 2017; Sanderson et al. 2014).

Under experimental conditions, we found that neither AuNps nor AuNpPEG have a cytotoxic effect on the cell lines tested here.

On the other hand, when the gold particle is the vehicle for the CisPt (AuNpPEGCisPt), the amount necessary to obtain the $\mathrm{IC}_{50}$ diminishes. In the case of the HeLa cells, it is necessary to use $69.2 \pm 3.2 \mu \mathrm{g} / \mathrm{mL}$ of CisPt and only $104.5 \pm 7.0 \mu \mathrm{g} / \mathrm{mL}$ of AuNpPEGCisPt. At first sight, it would seem that we use more CisPt. However, we must take into consideration that in the case of the HeLa cells, the AuNpPEGCisPt only contains approximately $7 \%$ of CisPt to obtain $\mathrm{IC}_{50}$. We used only $7.31 \mu \mathrm{g} / \mathrm{mL}$ of CisPt to obtain the same $\mathrm{IC}_{50}$. We observed the same tendency in the other cell lines (Table 2).

The synthesized nanoparticles must have a series of characteristics to be a valid alternative in the treatment of cancer. They must be harmless and not destroy normal cells. In our case, both AuNps and AuNpPEG comply with this requirement. AuNpPEG can therefore function as a vehicle for the CisPt. We have shown that AuNpPEGCisPt contains small amounts of CisPt. However, a smaller amount of CisPt is required, if it is attached to the AuNPEGCisPt, than the quantity required if $\mathrm{CisPt}$ is administered alone.

Previous work has shown that the Nps can suffer a series of inconveniences when they come into contact with a more complex system such as that of the human metabolism. Nps can be recognized by the immune system and become less effective due to a low bioavailability (Gustafson et al. 2015). Thus, we decided to functionalize our AuNps with PEG, which has been shown to help AuNps to gain solubility, evade the immune system and therefore increase their half-life (Li et al. 2014a, b). In our case, we have also observed an increase in the effectiveness of CisPt in which it diminishes the $\mathrm{IC}_{50}$, despite the fact that the AuNpPEGCisPt forms agglomerates. This phenomenon seems to support our position. Nps functionalized with PEG have the ability to bind to cell membranes and, in the case of AuNpPEGCisPt, because of its size, may permit it to cross the cell membrane. This would facilitate the arrival of CisPt inside the cell and its subsequent transportation to the nucleus, which is where this compound would take effect (Suk et al. 2016).

Reports show that cancer cells can reduce the effects of CisPt by binding to the metallothionine protein or glutathione (Huska et al. 2009). The CisPt ejects through the membrane (Ahmad 2017). By joining the CisPt to AuNpPEG, these proteins may no longer recognize the CisPt because it is inside the core-shell nanoparticle ( $\mathrm{Li}$ et al. 2014a, b; Zrazhevskiy et al. 2010). This is the reason why, in this investigation, we used much smaller amounts of CisPt (Brown et al. 2010). The AuNp, because of its size and polarity, helps the CisPt to cross the cellular membrane (Lin et al. 2010). In 
addition, AuNpPEG works as a masking system for the CisPt (Babu et al. 2013). CisPt is poorly soluble in water. One must suspend it in water to reach the nucleus of the cell. It seems that AuNpPEGCisPt improves its solubility (Johnstone et al. 2016).

The size of the AuNpPEGCisPt provides a large contact surface that facilitates its interaction with the genetic material of the cell (Wang et al. 2016). It also enhances the interaction of CisPt with the nitrogenous bases of DNA, intervening in the functions of cellular transcription and replication and causes cell death by apoptosis (Hall et al. 2007).

\section{Conclusions}

We developed a new methodology for the synthesis of a Gold core-shell type nanoparticle. It contains the anticancer CisPt within the shell and may facilitate transport across the cell membrane.

This in vitro study has determined that, if we use AuNps as the nanocarriers, the amount of CisPt necessary to cause the desired cytotoxic effect is less than the amount used in conventional cancer treatments.

These results show that the synthesized AuNpPEGCisPt is a good transport of CisPt. It decreases substantially the dose and has the same effect as the CisPt, so that the undesired side effects may decrease. The synthesized nanoparticles AuNps and AuNpPEG could be potential nanovehicles for the creation of theranostic nanoparticles.

\section{Material and method}

\section{Synthesis of nanoparticles}

We synthesized gold nanoparticles using the chemical reduction method (Turkevich et al. 1951). We cleaned the glass material with a solution of aqua regia at 1: $3\left(\mathrm{HNO}_{3}+3\right.$ $\mathrm{HCl}$ ). Afterward, we washed the material with $\mathrm{H}_{2} \mathrm{O}$ miliQ.

First, we heated $0.01 \mathrm{M}$ of $\mathrm{HAuCl}_{4} \cdot 3 \mathrm{H}_{2} \mathrm{O}$ [Gold (III) chloride trihydrate, sigma 520918] with miliQ water in a ratio of $1: 10$. We added sodium citrate $(18 \mathrm{~mL}$ of $38.88 \mathrm{mM}, \mathrm{S} 4641$ Sigma-Aldrich), boiled the mixture for $15 \mathrm{~min}$ with magnetic agitation, and let it cool down to $4{ }^{\circ} \mathrm{C}$ until use. Next, we functionalized AuNps with PEG and CisPt, adapting the method used by Sun et al. (2014).

We synthesized AuNpPEG by mixing $1 \mathrm{mg}$ of AuNps with $1 \mathrm{mg}$ of PEG (average Mn 3500. 757837 Aldrich) stirring the mixture for $18 \mathrm{~h}$ at room temperature. We eliminated the excess of PEG by centrifuging the mixture at $15000 \mathrm{rpm}$ for $15 \mathrm{~min}$ at $4{ }^{\circ} \mathrm{C}$.

We synthesized the AuNpPEGCisPt by mixing $1 \mathrm{mg}$ AuNps with $1 \mathrm{mg}$ of PEG and $1 \mathrm{mg}$ of CisPt (P4394 Sigma). The solutions remained in constant magnetic agitation for $18 \mathrm{~h}$ at room temperature. Later, we separated the PEG and CisPt that did not bind to the AuNps by centrifuging the product at $15,000 \mathrm{rpm}$ for $15 \mathrm{~min}$ at $4{ }^{\circ} \mathrm{C}$. We washed the synthesized nanoparticles with $\mathrm{H}_{2} \mathrm{O}$ miliQ on three occasions and stored them at $4{ }^{\circ} \mathrm{C}$ until use.

\section{Characterization of the gold nanoparticles}

We analyzed the size, microstructure, atomic concentration, chemical composition and morphology of the samples using scanning electron microscopy with energy dispersive spectroscopy (SEM/EDS), transmission electron microscopy (TEM), ultraviolet-visible 
light spectroscopy (UV/VIS) and Fourier transform infrared spectroscopy (FTIR). The dynamic light scattering (DLS) technique was employed to obtain the particle size and PDI values. Meanwhile, we measured Zeta Potential.

\section{Ultraviolate/visible spectroscopy (UV/VIS)}

We read the spectrometric absorbance of the synthesized nanoparticles by means of an Ultraviolet-Visible Spectrophotometer (UV-Vis) SPECTROstar nano BMG labtech spectrophotometer. $50 \mu \mathrm{L}$ of nanoparticles was selected and deposited in a quartz cuvette. We recorded the optical absorbance at a wavelength range between 200 and $800 \mathrm{~nm}$.

\section{Fourier transform infrared spectroscopy (FTIR)}

We analyzed the samples in solution by FTIR spectroscopy, using two Perkin Elmer spectrometers. $50 \mu \mathrm{L}$ of the nanoparticles were placed in the universal ATR plate. It was necessary to establish good contact with the sample to carry out the reading. The wavenumber interval used for this analysis was from 4000 to $400 \mathrm{~cm}^{-1}$, at a resolution of $1.0 \mathrm{~cm}^{-1}$.

\section{Transmission electron microscopy (TEM)}

For the analysis of the microstructure, we placed the AuNps, AuNpPEG or AuNpPEGCisPt on a formvar coated onto copper grids and examined them with a Transmission Electron Microscope Philips Tecnai-10, at an accelerating rate of $100 \mathrm{kV}$. We determined the size of the nanoparticles using the program Soft Imaging System analySIS 3.0.

\section{Scanning electron microscopy with energy dispersive spectroscopy (SEM/EDS)}

For the analysis of the microstructure, chemical composition and atomic concentration, we placed the nanoparticles on a $7 \mathrm{~mm} \times 12 \mathrm{~mm}$ aluminum sample holder and loaded it into the vacuum chamber of a scanning electron microscope Hitachi SU5000 SEM. The distance between the samples and the electron source was $10 \mathrm{~cm}$. An electron beam with an energy of $20 \mathrm{keV}$ impinged on the sample. We determined the size of the nanoparticles using the program Image J (version 1.52a).

\section{Hydrodynamic size and zeta potential}

To characterize the nanoparticles, we measured their size, distribution (Polydispersity Index, PDI) and zeta potential. The dynamic light scattering (DLS) technique was employed to obtain the particle size and PDI values. Meanwhile, Zeta Potential was measured by Velocimetry Laser Doppler (VLS). AuNps, AuNpPEG and AuNpPEGCisPt were resuspended in $\mathrm{H}_{2} \mathrm{O}$, culture medium DMEM with bovine fetal serum (serum) at $10 \%$ and DMEM and culture medium DMEM without bovine fetal serum (w/o serum). The measurements were performed using a Malvern Instrument ZS90; Malvern, UK Results are reported in terms of average \pm standard deviation (SD).

\section{Cell culture}

We cultivated three cancer cell lines. These were cervical cancer (HeLa), gastric cancer (AGS) and neuroblastoma (C6). All the cell lines were obtained from American Type Culture Collection (ATCC, Rockville, MD, USA). 
We used the cells obtained to interact with the nanoparticles and determine $\mathrm{IC}_{50}$. The cells grew for $24 \mathrm{~h}$ in $25 \mathrm{~cm}^{2}$ culture bottles with DMEM medium (Dulbecco's Modified Eagle Medium, Thermo Fisher 11,995-065) supplemented with $10 \%$ fetal bovine serum (Thermo Fisher 16000-044), 1\% antibiotic (10,000 U/mL penicillin and 10,000 $\mu \mathrm{g} / \mathrm{mL}$ streptomycin, Thermo Fisher 15140122$)$ at $37^{\circ} \mathrm{C}$ and $5 \% \mathrm{CO}_{2}$.

\section{The in vitro tests for the determination of the $\mathrm{IC}_{\mathbf{5 0}}$ (Fig. 6)}

We carried out the assays in triplicate in a 96-well plate. In each well, we deposited $4 \times 10^{4}$ cells and allowed them to adapt for $24 \mathrm{~h}$ at $37{ }^{\circ} \mathrm{C}$ and $5 \% \mathrm{CO}_{2}$. Subsequently, we added increasing amounts of AuNps, AuNpPEG and AuNpPEGCisPt (62.5, 125, 250, 500 and $1000 \mu \mathrm{g} / \mathrm{mL}$ ). We left them incubating for $24 \mathrm{~h}$ at $37^{\circ} \mathrm{C}$ and $5 \% \mathrm{CO}_{2}$. To determine the percentage of viability and the $\mathrm{IC}_{50}$, we added $10 \mu \mathrm{L}$ of a solution of 3-(4,5-dimethylthiazol-2-yl)-2,5-diphenyltetrazolium bromide (MTT, M5655 Sigma) at a concentration of $5 \mathrm{mg} / \mathrm{mL}$ to each well and incubated them for $4 \mathrm{~h}$ at $37{ }^{\circ} \mathrm{C}$ and $5 \% \mathrm{CO}_{2}$. Next, we added $100 \mu \mathrm{L}$ of dimethyl sulfoxide (DMSO, D2650 Sigma). Finally, we read the plate in a spectrophotometer at $570 \mathrm{~nm}$ (SPECTROstar nano BMG labtech.). We determined the $\mathrm{IC}_{50}$ using the Graph Pad Prism version 6.00 for Windows, GraphPad Software, La Jolla California USA, http://www.graphpad.com. We included the following controls to the feasibility test: a positive control with CisPt (at a concentration of 62.5, 125, 250, 500 and $1000 \mu \mathrm{g} / \mathrm{mL}$ ) and a negative control without treatment. We also carried out UV/ VIS to determine the wavelengths of the cancer cells and clarify the problem of possible overlap with the wavelengths of the different synthesized Nps and the MTT metabolism product (formazan). We would thus avoid overvalued figures. We placed an aliquot of cancer cells, Nps or formazan in a cuvette at a concentration (optical density, OD) of between 0.8 and 1 . We measured the absorbance peaks of the cells, Nps and formazan. Subsequently, the following combinations were made: Cells + AuNps, Cells + AuNpPEG and Cells + AuNpPEGCisPt.

\section{Statistical analysis}

We presented the data as means \pm SEM from at least three independent experiments and compared by one-way analysis of variance (ANOVA) followed by multiple

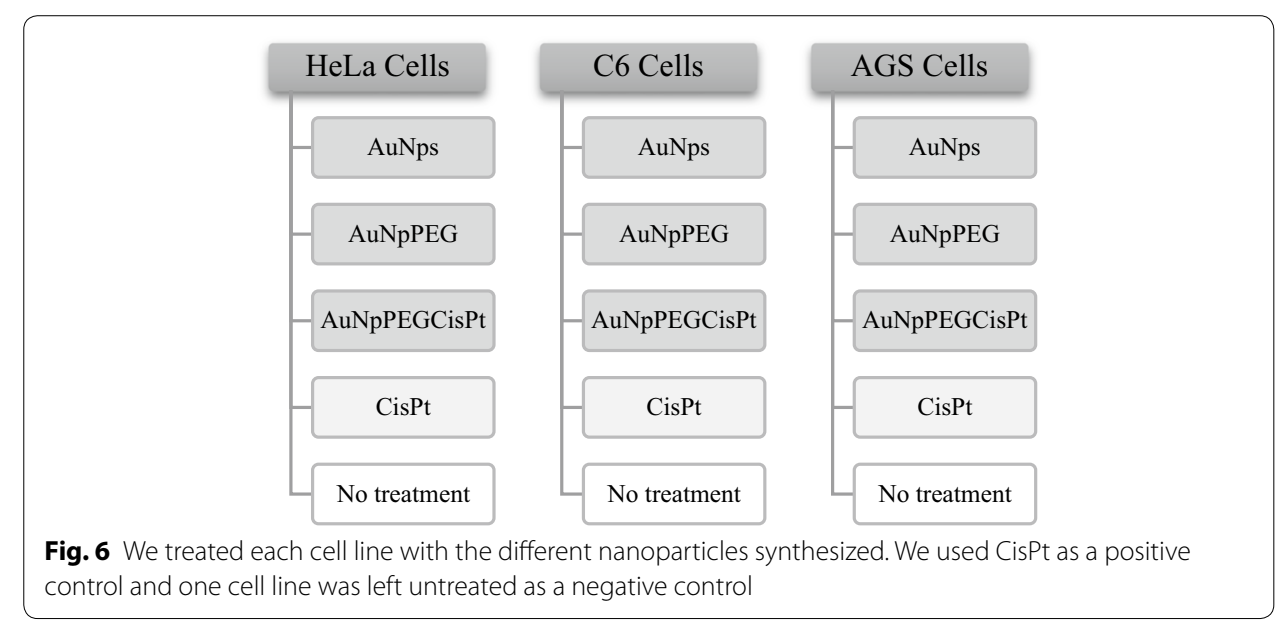


comparison analysis averages Tukey. $P$ value less than 0.05 was considered significant. We carried out statistical analyses with the GraphPad Prism version 6.00 for Windows, GraphPad Software, La Jolla California USA, http://www.graphpad.com.

\section{Supplementary information}

Supplementary information accompanies this paper at https://doi.org/10.1186/s12645-020-00060-w.

Additional file 1. Ultraviolet-visible light spectroscopy of HeLa cells and synthesized nanoparticles.

\section{Abbreviations}

${ }^{\circ} \mathrm{C}$ : Degrees celsius; ANOVA: Analysis of variance; ATCC: American type culture collection; ATR: Attenuated total reflectance; AuNpPEG: Gold nanoparticle functionalized with polyethylene glycol; AuNpPEGCisPt: Gold nanoparticle functionalized with polyethylene glycol and cisplatin; AuNps: Gold nanoparticles; CisPt: Cisplatin; $\mathrm{cm}^{-1}$ : Reciprocal centimeter or wavenumber; DLS: Dynamic light scattering; DMEM: Dulbecco's modified Eagle medium; DMSO: Dimethylsulfoxide; DNA: Deoxyribonucleic acid; EDS: Energy dispersion spectroscopy; FTIR: Fourier transform infrared spectroscopy; g: Grams; $\mathrm{HCl}$ : Hydrochloric acid; $\mathrm{HNO}_{3}$ : Nitric acid; $\mathrm{IC}_{50}$ : Half maximal inhibitory concentration; keV: Kiloelectron voltage; $\mathrm{M}$ : Molar concentration; mg: Milligrams; min: Minutes; $\mu \mathrm{g}$ : Micrograms; mL: Microliter; mL: Milliliter; mm: Millimeters; mM: Millimolar concentration; MTT: 3-(4,5-dimethylthiazol-2-yl)-2,5-diphenyltetrazolium bromide; mV: Millivolts; nm: Nanometers; Nps: Nanoparticles; OD: Optical density; PDI: Polydispersity index; PEG: Polyethylene glycol; rpm: Revolutions per minute; SD: Standard deviation; SEM/EDS: Scanning electron microscopy/energy dispersive X-ray spectroscopy; TEM: Electronic transmission microscopy; UVNIS: Ultraviolet-visible light spectroscopy; VLS: Velocimetry Laser Doppler; WHO: World Health Organization.

\section{Acknowledgements}

The authors would like to thank: Michael Shea (Dept. Education and Communication UAM-X) for translating this paper. The authorities of the Universidad Autónoma Metropolitana, Unidad Xochimilco for their support. The authorities of the Unidad de Investigación en Biomedicina (UBIMED) of the FES Iztacala, Universidad Nacional Autónoma de México for their support. We thank Alma Delia Hernández Pérez, Ph.D. Gerardo Leyva and M.Sc. Edgar Ortiz Benitez for helpful suggestion and technical support. Conacyt groups: BCSC, LQCLBC and BCAUyCSM. To my Becaria.

\section{Authors' contributions}

All authors listed have made substantial, direct, and intellectual contributions to the work discussed in this publication. GLMA devised the overall project, prepared the nanoparticles, performed DLS, DSI, Potential Z, UVNVIS, FTIR, cell culture, MTT experiments and analyzed the data. GCEM performed the experiments DLS, DSI, Potential $Z$, cell culture and analyzed the data; CSC performed UV/VIS experiments of controls and analyzed the data; HPJF and PI performed the EDS/ SEM analysis and interpreted the results. OTJJ participated in the synthesis of nanoparticles. HGO performed TEM, DLS, DSI and Potential $Z$ analysis and interpreted the results. All authors read and approved the final manuscript.

\section{Funding}

The authors would like to thank: The Program Cátedras CONACYT project 1027 and 3035. CONACYT for J.J. OlivaresTrejo's scholarship to make a sabbatical year (Convocatoria Estancia Sabática Nacional 2019-1) and The Consejo Nacional de Ciencia y Tecnología (CONACYT) for supporting this research (Grant Number 269533).

\section{Availability of data and materials \\ Not applicable.}

\section{Ethics approval and consent to participate}

Not applicable.

\section{Consent for publication}

All the authors have given consent for publication.

Competing interests

The authors declare that they have no competing interests.

\section{Author details}

1 Unidad de Oncología Torácica., CONACYT-Instituto Nacional de Cancerología, Cátedra-CONACYT, Avenida San Fernando 22, Col. Belisario Domínguez Secc. 16, 14080 Ciudad de México, México. ${ }^{2}$ Departamento de Atención a la Salud, Universidad Autónoma Metropolitana, Unidad Xochimilco, Calzada del Hueso 1100, Col. Villa Quietud, Delegación Coyoacán, 04960 Ciudad de México, México. ${ }^{3}$ Institute of Engineering and Technology, Universidad Autónoma de Ciudad Juárez, Av. del Charro 450, Col. Romero Partido, 32310 Juárez, Chihuahua, México. ${ }^{4}$ National Council of Science and Technology (CONACYT), Institute of Engineering and Technology, Universidad Autónoma de Ciudad Juárez, Av. del Charro 450, Col. Romero Partido, 32310 Juárez, Chihuahua, México. ${ }^{5}$ Posgrado en Ciencias Genómicas, Universidad Autónoma de la Ciudad de México, Av. San Lorenzo 290, Col. Del Valle, 03100 Ciudad de México, México. ${ }^{6}$ Laboratorio de Microscopia Electrónica, Instituto Nacional de Rehabilitación, Calzada México Xochimilco No. 289, Colonia Arenal de Guadalupe, Alcaldía Tlalpan, 14389 Ciudad de México, México. 
Received: 2 December 2019 Accepted: 13 March 2020

Published online: 30 March 2020

\section{References}

Ahmad S. Kinetic aspects of platinum anticancer agents. Polyhedron. 2017;138(14):109-24. https://doi.org/10.1016/j. poly.2017.09.016.

Alcantar NA, Aydil ES, Israelachvili JN. Polyethylene glycol-coated biocompatible surfaces. J Biomed Mater Res. 2000;51(3):343-51. https://doi.org/10.1002/10974636(20000905)51:3\%3C343:AIDJBM7\%3E3.0.CO;2-D.

Alkilany AM, Murphy CJ. Toxicity and cellular uptake of gold nanoparticles: what we have learned so far. J Nanopart Res. 2010;12(7):2313-33. https://doi.org/10.1007/s11051-010-9911-8.

Altunbek M, Culha M. Influence of plasmonic nanoparticles on the performance of colorimetric cell viability assays. Plasmonics. 2017;12:1749-60. https://doi.org/10.1007/s11468-016-0442-8.

Astolfi L, Ghiselli S, Guaran V, Chicca M, Simoni E, Olivetto E, Lelli G, Martini A. Correlation of adverse effects of cisplatin administration in patients affected by solid tumours: a retrospective evaluation. Oncol Rep. 2013;29(4):1285-92. https://doi.org/10.3892/2For.2013.2279.

Babu A, Templeton AK, Munshi A, Ramesh R. Nanoparticle-based drug delivery for therapy of lung cancer: progress and challenges. J Nanomater. 2013;2013:11. https://doi.org/10.1155/2013/863951.

Balice V, Theophanides T. I. R. Spectra of platinum (ii) histidine complexes. J Inorg Nacl Chem. 1970;32:1237-40. https:// doi.org/10.1016/0022-1902(70)80120-8.

Baowan D, Cox B, Hilder T, Hill J, Thamwattana N. Modelling and mechanics of carbon-based nanostructured materials. USA:William Andrew Publishing; 2017.

Botha TL, James TE, Wepener V. Comparative aquatic toxicity of gold nanoparticles and ionic gold using a species sensitivity distribution approach. J Nanomat. 2015;2015:1-16. https://doi.org/10.1155/2015/986902.

Brown SD, Nativo P, Smith JA, Stirling D, Edwards PR, Venugopal B, Flint DJ, Plumb JA, Graham D, Wheate NJ. Gold nanoparticles for the improved anticancer drug delivery of the active component of oxaliplatin. J Am Chem Soc. 2010;132(13):4678-84. https://doi.org/10.1021/ja908117a.

Couto C, Vitorino R, Daniel-da-Silva AL. Gold nanoparticles and bioconjugation: a pathway for proteomic applications. Crit Rev Biotechnol. 2016;37(2):238-50. https://doi.org/10.3109/07388551.2016.1141392.

Craig GE, Brown SD, Lamprou DA, Graham D, Wheate NJ. Cisplatin-tethered gold nanoparticles that exhibit enhanced reproducibility, drug loading, and stability: a step closer to pharmaceutical approval? Inorg Chem. 2012;51 (6):34907. https://doi.org/10.1021/ic202197g.

Danaei M, Dehghankhold M, Ataei S, Hasanzadeh Davarani F, Javanmard R, Dokhani A, Khorasani S, Mozafari MR. Impact of particle size and polydispersity index on the clinical applications of lipidic nanocarrier systems. Pharmaceutics. 2018. https://doi.org/10.3390/pharmaceutics10020057.

Guerrini L, Alvarez-Puebla RA, Pazos-Perez N. Surface modifications of nanoparticles for stability in biological fluids. Materials. 2018;11(7):E1154. https://doi.org/10.3390/ma11071154.

Gustafson HH, Holt-Casper D, Grainger DW, Ghandehari H. Nanoparticle uptake: the phagocyte problem. Nano Today. 2015;10(4):487-551. https://doi.org/10.1016/j.nantod.2015.06.006.

Ha TH, Jeong JY, Chung BH. Immobilization of hexa-arginine tagged esterase onto carboxylated gold nanoparticles. Chem Commun. 2005;21(31):3959-61. https://doi.org/10.1039/B504184H.

Hall MD, Mellor HR, Callaghan R, Hambley TW. Basis for design and development of platinum(IV) anticancer complexes. J Med Chem. 2007;50(15):3403-11. https://doi.org/10.1021/jm070280u.

Huska D, Fabrik I, Baloun J, Adam V, Masarik M, Hubalek J, Vasku A, Trnkova L, Horna A, Zeman L, Kizek R. Study of interactions between metallothionein and cisplatin by using differential pulse voltammetry brdickás reaction and quartz crystal microbalance. Sensors. 2009:9(3):1355-69. https://doi.org/10.3390/2Fs90301355.

Ji X, Song X, Li J, Bai Y, Yang W, Peng X. Size control of gold nanocrystals in citrate reduction: the third role of citrate. J Am Chem Soc. 2007;129(45):13939-48. https://doi.org/10.1021/ja074447k.

Johnstone TC, Suntharalingam K, Lippard SJ. The next generation of platinum drugs: targeted Pt(II) agents, nanoparticle delivery, and Pt(IV) prodrugs. Chem Rev. 2016;116(5):3436-86. https://doi.org/10.1021/2Facs.chemrev.5b00597.

Kaushik A, Solanki PR, Ansari AA, Sumana G, Ahmad S, Malhotra BD. Iron oxide-chitosan nanobiocomposite for urea sensor. Sens Actuator B Chem. 2009;138:572-80. https://doi.org/10.1016/j.snb.2009.02.005.

Kuo TR, Wu CL, Hsu CH, Lo W, Chiang SJ, Lin SJ, Dong CY, Chen CC. Chemical enhancer induced changes in the mechanisms of transdermal delivery of zinc oxide nanoparticles. Biomaterials. 2009:30:3002-8. https://doi.org/10.1016/j. biomaterials.2009.02.003.

Li D, Li Q, Hao X, Zhang Y, Zhang Z, Li C. Assembled core-shell nanostructures of gold nanoparticles with biocompatible polymers toward biology. Curr Top Med Chem. 2014a;14(5):595-616. https://doi.org/10.2174/156802661466614 0118204000.

Li Y, Kröger M, Liu WK. Endocytosis of PEGylated nanoparticles accompanied by structural and free energy changes of the grafted polyethylene glycol. Biomaterials. 2014b;35(30):8467-78. https://doi.org/10.1016/j.biomaterials.2014.06.032.

Lin J, Zhang H, Chen Z, Zheng Y. Penetration of lipid membranes by gold nanoparticles: insights into cellular uptake, cytotoxicity, and their relationship. ACS Nano. 2010;28(9):5421-9. https://doi.org/10.1021/nn1010792.

López GTM (2013) Nanomedicina Catalítica: Ciencia y Cáncer. Ed. Arkhé. Universidad Autónoma Metropolitana, Unidad Xochimilco. Fondo de Cooperación Internacional en Ciencia y Tecnología Unión Europea-México.

Lowry GV, Hill RJ, Stacey H, Rawle AF, Hendren Ch, Klaessig F, Nobbmann U, Sayre P, Rumble J. Guidance to improve the scientific value of zeta-potential measurements in nanoEHS. Environ Sci: Nano. 2016;3:953-65. https://doi. org/10.1039/C6EN00136J.

Ma P, Xiao H, Li Ch, Dai Y, Cheng Z, Hou Z, Lin J. Inorganic nanocarriers for platinum drug delivery. Mater Today. 2015;18(10):554-64. https://doi.org/10.1016/j.mattod.2015.05.017. 
Moore TL, Rodriguez-Lorenzo L, Hirsch V, Balog S, Urban D, Jud C, Rothen-Rutishauser B, Lattuada M, Petri-Fink A. Nanoparticle colloidal stability in cell culture media and impact on cellular interactions. Chem Soc Rev. 2015;44(17):6287305. https://doi.org/10.1039/c4cs00487f.

Nath RD, Goswami R, Pal A. Nanomaterial and toxicity: what can proteomics tell us about the nanotoxicology? Xenobiotica. 2017;47(7):632-43. https://doi.org/10.1080/00498254.2016.1205762.

Ortiz-Benitez EA, Carrillo-Morales M, Velázquez-Guadarrama N, Fandiño-Armas J, de Olivares-Trejo J. Inclusion bodies and pH lowering: as an effect of gold nanoparticles in Streptococcus pneumoniae. Metallomics. 2015;7(7):1173-9. https://doi.org/10.1039/C5MT00044K.

Rajath O, Pardhasaradhi M, Khaggeswar B, Lavanya K, Mahendran B. Characterization of vitamin-cisplatin-loaded chitosan nano-particles for chemoprevention and cancer fatigue. J Microencapsul. 2015;32(6):578-88. https://doi. org/10.3109/02652048.2015.1065921.

Sánchez E, López T, Gómez R, Bokhimi X, Morales A, Novaro O. Synthesis and characterization of sol-gel Pt/TiO 2 . Catal J Solid State Chem. 1996;122:309-14. https://doi.org/10.1007/s10450-011-9330-x.

Sanderson BJS, Lam R, Alharthia J, Shapterb J. The potential of gold nanoparticle conjugates to kill cancer cells in culture. Procedia Eng. 2014;92:26-9. https://doi.org/10.1016/j.proeng.2013.10.004.

Suk JS, Xu Q, Kim N, Hanes J, Ensign LM. PEGylation as a strategy for improving nanoparticle-based drug and gene delivery. Adv Drug Deliv Rev. 2016;99(Pt A):28-51. https://doi.org/10.1016/j.addr.2015.09.012.

Sun Y, Sai H, Von Stein F, Riccio M, Wiesner U. Water-based synthesis of ultrasmall pegylated gold-silica core-shell nanoparticles with long-term stability. Chem Mater. 2014;26(18):5201-7. https://doi.org/10.1021/cm501348r.

Turkevich J, Stevenson PL, Hillier J. A study of the nucleation and growth process in the synthesis of colloidal gold. Discuss Faraday Soc. 1951;11:55-75. https://doi.org/10.1039/DF9511100055.

Vijaya K, Ganesan S. Preparation and characterization of gold nanoparticles with different capping agents. Int J Green Nanotechnol. 2011;3(1):47-55. https://doi.org/10.1080/19430892.2011.574538.

Wang P, Wang X, Wang L, Hou X, Liu W, Chen C. Interaction of gold nanoparticles with proteins and cells. Sci Technol Adv Mater. 2015;16(3):034610. https://doi.org/10.1088/1468-6996/16/3/034610.

Wang X, Hu X, Li J, Russe AC, Kawazoe N, Yang Y, Chen G. Influence of cell size on cellular uptake of gold nanoparticles. Biomater Sci. 2016;4(6):970-8. https://doi.org/10.1039/c6bm00171h.

World Health Organization (WHO). http://www.who.int/en/news-room/fact-sheets/detail/cancer. Accessed 29 Enero 2020.

Zrazhevskiy P, Sena M, Gao X. Designing multifunctional quantum dots for bioimaging, detection, and drug delivery. Chem Soc Rev. 2010;39(11):4326-54. https://doi.org/10.1039/2Fb915139g.

\section{Publisher's Note}

Springer Nature remains neutral with regard to jurisdictional claims in published maps and institutional affiliations.

- fast, convenient online submission

- thorough peer review by experienced researchers in your field

- rapid publication on acceptance

- support for research data, including large and complex data types

- gold Open Access which fosters wider collaboration and increased citations

- maximum visibility for your research: over 100M website views per year

At BMC, research is always in progress.

Learn more biomedcentral.com/submissions 